88 FACTORS CONCERNING SCHOOL-BASED POLICY AND THE IMPLEMENTATION OF THE NATA-IATF PRESEASON HEAT ACCLIMATIZATION GUIDELINES IN HIGH SCHOOL FOOTBALL

'Zachary Kerr, ${ }^{2}$ Melissa Kay, ${ }^{1}$ Aliza Nedimyer, ${ }^{1}$ Avinash Chandran, ${ }^{3}$ Riana Pryor, ${ }^{4}$ Douglas Casa, 'Johna Register-Mihalik. 'University of North Carolina at Chapel Hill; ${ }^{2}$ University of Southern Mississippi; ${ }^{3}$ SUNY Buffalo; ${ }^{4}$ University of Connecticut

10.1136/injuryprev-2020-savir.107

Statement of Purpose Our study aimed to identify factors concerning school-based policy that were associated with the implementation of the National Athletic Trainers' Association (NATA) Inter-Association Task Force (IATF) preseason heat acclimatization guidelines to prevent exertional heat illness (EHI).

Methods/Approach Semi-structured phone interviews regarding implementing the NATA-IATF guidelines were conducted with a purposive sample of 33 US high school football athletic trainers (ATs; 16 male/17 female; mean age $=36.4 \pm 12.5$ years). Interviews were transcribed verbatim. Four progressive stages in the consensual qualitative research tradition were utilized to create a codebook. Study personnel coded interviews and held consensus meetings to resolve coding discrepancies.

Results Participants highlighted themes related to school-based policy in implementing the NATA-IATF guidelines. First, participants discussed using the NATA-IATF guidelines as primary sources to initiate and inform school-based policy; however, policy was noted needing updates to account for current EHI prevention/management best practices. Second, participants noted state laws and state-specific athletic associations (not school-based policy) were the primary resource for EHI prevention among administrators and coaches; however, these resources did not always align with best practices, creating frustration for ATs. Third, participants emphasized needing policy that included accountability and enforcement, particularly for coaches; most looked to state athletic associations and administrators for such support. Last, school-based policy seemed more beneficial when in schools located in states where NATA-IATF guidelines were mandated and when in warmer areas where there was higher perceived EHI risk.

Conclusions Gaps existing in school-based policy may affect the implementation of the NATA-IATF guidelines. Policy should be continually updated, align with best practices, and consider accountability and enforcement. Future research should also identify factors associated with disparities in implementation.

Significance/Contribution to Injury and Violence Prevention Science School-based policy may be helpful in facilitating the implementation of the NATA-IATF guidelines and thus, increasing their effectiveness in reducing EHI.

\section{DESCRIPTIVE EPIDEMIOLOGY OF LITHIUM BATTERY THERMAL RUNAWAY-RELATED INJURIES IN THE UNITED STATES FROM 2014-2018}

E Andrew Kapp, Rachael Beer, David Wroth. Underwriters Laboratory Inc

\subsection{6/injuryprev-2020-savir.108}

Objective Lithium batteries have the potential to overheat and explode, causing injury to users of consumer devices like cell phones, laptops, and electronic nicotine delivery systems (ENDS). Given the widespread use of such products, it is critical to further understand how often such injuries occur and the circumstances surrounding them.

Methods The CPSC monitors consumer product-related injuries using their National Electronic Injury Surveillance System (NEISS), which compiles reports from a representative sample of US emergency departments. Hospitals submit patient information including circumstances of injury, diagnosis, body part injured, and demographic information. To identify cases related to lithium batteries, NEISS data were filtered using diagnosis codes, product codes, and word stems in text narratives.

Results From 2014-2018, there were 138 recorded emergency department visits associated with malfunctioning lithium batteries. Implementing the complex survey design of NEISS data, this translates to an estimate of 5,000 such injuries nationwide. Visits occurred mainly due to ENDS (48\%) and cell phones (23\%). 81\% of incidents involved men and boys. $20 \%$ of patients were admitted to the hospital. Injuries were commonly caused by products exploding (64\%) or overheating (35\%) and characterized by being kept in one's pocket $(39 \%)$ or being in use $(17 \%)$ at the time of injury.

Conclusions This study provides valuable information on the number and type of lithium battery-related injuries treated in US Emergency Departments. Identifying the most common circumstances of injuries will inform future research on the development of safety features in these commonly used devices, as well as potential opportunities for consumer education.

\section{ESTABLISHING THE EFFECTIVENESS OF A STORYBOOK FOR TEACHING HOME SAFETY TO PRESCHOOL CHILDREN}

Barbara Morrongiello. University of Guelph - Canada

\subsection{6/injuryprev-2020-savir.109}

Statement of Purpose Unintentional injuries are a serious concern for young children, frequently resulting in disability and death. Safety interventions that target the child as an agent of change are needed, particularly because parents cannot constantly supervise. The present study examined whether a storybook can educate about home hazards and reduce the hazarddirected risk behaviours of children aged 3.5 to 5.5 years.

Methods Preschoolers were randomly assigned to the control condition (a storybook about healthy eating) or the intervention condition (a storybook about home hazards) and were required to read the assigned storybook with their mother for four weeks.

Results Comparing children's pre- and post-intervention knowledge and risk behaviours indicated that children in the intervention condition were able to identify more hazards, provide more comprehensive explanations, and demonstrate less risky behaviours, in comparison to those in the control group. Hence, the storybook improved both safety knowledge and behaviors.

Conclusion Together, the findings suggest that a storybook can be an effective resource in educating young children about home safety and promoting safety practices that are likely to reduce risk of injury.

Significance The findings indicate that engaging children in reading a storybook about home hazards with their parents can not only increase their knowledge but also reduce their hazard-directed risk behaviors at home. 